\title{
On the Extension of Importance Measures to Complex Components
}

\author{
Yves Dutuit $^{\mathrm{a}}$ Antoine Rauzy ${ }^{\mathrm{b}}$ \\ aMember of Total "Professeurs Associés", Bordeaux, France, yves.dutuit@sfr.fr \\ ${ }^{\mathrm{b}}$ Chair Blériot-Fabre "Dependable Embedded Systems", Centrale-Supélec, Paris, \\ France, Antoine.Rauzy@ecp.fr
}

\begin{abstract}
Importance Measures are indicators of the risk significance of the components of a system. They are widely used in various applications of Probabilistic Safety Analyses, off-line and on-line, in decision making for preventive and corrective purposes, as well as to rank components according to their contribution to the global risk. They are primarily defined for the case the support model is a coherent fault tree and failures of components are described by basic events of this fault tree.

In this article, we study their extension to complex components, i.e. components whose failures are modeled by a gate rather than just a basic event. Although quite natural, such an extension has not received much attention in the literature. We show that it raises a number of problems. The Birnbaum Importance Measure and the notion of critical states concentrate these difficulties. We present alternative solutions for the extension of these notions. We discuss their respective advantages and drawbacks.

This article gives a new point of view on the mathematical foundations of Importance Measures and helps to clarify their physical meaning.
\end{abstract}

Key words: Fault Trees, Importance Measures, Complex Components

\section{Introduction}

Importance Measures are indicators of the risk significance of the components of a system. They are widely used in various applications of Probabilistic Safety Analyses, off-line and on-line, in decision making for preventive and corrective purposes, as well as to rank components according to their contribution to the global risk. Presentations of these indicators and discussions about their mathematical properties and their physical interpretations can be found for instance in References [1-12]. 
Importance Measures are primarily defined for the case the support model is a coherent Fault Tree and failures of components are represented by basic events of this fault tree. In this article, we study their extension to complex components, i.e. to components whose failure are modeled by a gate and not just by a Basic Event. Although quite natural, this extension has not received much attention in the literature (see however $[13,14]$ ).

We proceed in two steps. First, we revisit definitions of the main Importance Measures and we show that, in the case of simple components, each of them characterizes the probability of a set of minterms, i.e. of a set of global states of the system under study. Namely,

- The states in which both the component and the system are failed, as for the Diagnostic Importance Factor and the Risk Achievement Worth.

- The states in which the system is failed but the component is working, as for the Risk Reduction Worth.

- The critical states, i.e. states in which failing/repairing the component suffices to repair/fail the system, as for the Birnbaum Importance Measure (also called Marginal Importance Factor) and the Critical Importance Measure.

This new way of defining Importance Measures via minterms does not mean that they need to be calculated via minterms. Calculations can actually be still performed by means of Minimal Cutsets or Binary Decision Diagrams. Its interest stands in the soundness of mathematical definitions, the independence of any calculation means and the simplicity of physical interpretations.

Second, we show that this nice correspondence between the probabilistic definition and the minterm interpretation does not hold for complex components. The Birnbaum Importance Measure and the notion of critical states concentrate the difficulties.

So far, complex components have been studied in the literature only via the extension of Importance Measures to groups of (simple) components (see e.g. $[15,6,16,17,14,18,19,10,12])$. Several authors showed already that the definition of the Birnbaum Importance Measure in terms of a partial derivative is not suitable for groups of components (see e.g. [20,21]). They proposed therefore to define the Birnbaum Importance Measure as the difference between the conditional probability that the system is failed given that all components of the group are failed and the conditional probability that the system is failed given that none of the components of the group is failed. This definition is actually equivalent to the partial derivative one in the case the group is reduced to a single component. It could be applied to complex components as well, as proposed for instance by Sutter [14]. We show however that this indicator is much too coarse. First, it does not allow to distinguish components 
with different structure functions (a parallel sub-system would be evaluated the same way as a series sub-system). Second, it leads to consider as critical states states in which the system is failed but the component is working. We show that finer extensions can be defined, but necessarily to the price of loosing the correspondence between the probabilistic definition and the minterm interpretation.

This article contributes therefore to establish more firmly the mathematical foundations of Importance Measures and to clarify their physical interpretation. It also gives hints to tool developers about which indicators are worth to calculate from a safety model.

The remainder of this article is organized as follows.

- Section 2 introduces basic definitions and properties. It gives a formal definition for the notion of coherence and critical states.

- Section 3 revisits definitions and interpretations of Importance Measures in the case the support model is a coherent Fault Tree and failures of components are represented by Basic Events.

- Section 4 discusses extensions of Importance Measures to complex components and groups of components.

- Finally, section 5 concludes the article.

\section{Basic Definitions and Properties}

\subsection{Boolean Formulas and Minterms}

Throughout this article we consider Boolean formulae (Fault Trees) built over a denumerable set $\mathcal{E}$ of variables and the usual connectives "." (and), "+"" (or) and "-" (not). Variables are also called Basic Events.

We use upper case letters $E, A, B, C$, possibly with subscripts, to denote Basic Events.

We use lower case letters $s, t, c$, possibly with subscripts, to denote Boolean formulae. We denote by $\operatorname{var}(s)$ the variables occurring in the formula $s$.

Let $s$ be a Boolean formula. A variable assignment of $s$ is a function from $\operatorname{var}(s)$ into $\{0,1\}$ (0 and 1 stand respectively for False and True). Variable assignments are lifted-up as usual into functions from formulae into $\{0,1\}$ using the truth tables of connectives. A Boolean formula $s$ is interpreted as the Boolean function $\llbracket s \rrbracket$, i.e. as the function from variable assignments of $s$ 
into 0,1 , defined as follows: for any variable assignment $\sigma$ of $s, \llbracket s \rrbracket(\sigma)=1$ if $\sigma(s)=1$ and 0 otherwise.

In this article, we do not need to distinguish between syntax and semantics. Therefore, we shall assimilate the Boolean formula $s$ with its semantics $\llbracket s \rrbracket$.

A literal is either a variable $E$ or its negation $\bar{E}$. We use upper case letters $L$, $I, J$, possibly with subscripts to denote literals. We denote by $\bar{L}$ the opposite of a literal $L$, given that $\overline{\bar{L}} \equiv L$. Let $\mathcal{L}$ be a set of literals, we denote by $\overline{\mathcal{L}}$ the set of negations of literals of $\mathcal{L}$, i.e. $\{\bar{L} ; L \in \mathcal{L}\}$.

A product is a conjunct of literals that does not contain both a variable and its negation. Let $s$ be a Boolean formula. A minterm of $s$ is a product that contains a literal built over each variable of $\operatorname{var}(s)$. We use lower case Greek letters $\sigma, \tau \pi, \rho$, possibly with subscripts, to denote products and minterms. We denote as Minterms $(\mathcal{E})$ the set of $2^{|\mathcal{E}|}$ minterms that can be built over a set of Basic Events $\mathcal{E}$.

There is a one-to-one correspondence between variable assignments and minterms (and therefore between Boolean functions and sets or sums of minterms): the variable assignment $\sigma$ one-to-one corresponds with the minterm $\pi$ such that $\pi$ contains the positive literal $E$ if $\sigma(E)=1$ and the negative literal $\bar{E}$ if $\sigma(E)=0$. It follows that any Boolean formula $s$ is equivalent to the set of minterms $\pi$ such that $\pi(s)=1$. Minterms of $\operatorname{Minterms}(\mathcal{E})$ are the atoms of the Boolean algebra built over $\mathcal{E}$.

For the sake of the convenience, we shall use a set theory notation, i.e. we shall note $\pi \in s$ when $\pi(s)=1$ and $\pi \notin s$ when $\pi(s)=0$. Note also that $\pi \notin s$ if and only if $\pi \in \bar{s}$.

Example (Minterms): As an illustration, consider the two formulae $s_{1}=$ $A . B+A . C+B . C$ and $s_{2}=A . B+\bar{A} . C$. The minterms of $s_{1}$ and $s_{2}$ are as follows.

$$
\begin{aligned}
& s_{1} \equiv A \cdot B \cdot C+A \cdot B \cdot \bar{C}+A \cdot \bar{B} \cdot C+\bar{A} \cdot B \cdot C \\
& s_{2} \equiv A \cdot B \cdot C+A \cdot B \cdot \bar{C}+\bar{A} \cdot B \cdot C+\bar{A} \cdot \bar{B} \cdot C
\end{aligned}
$$

From a more practical perspective, assuming that the plant under study is modeled by a Fault Tree, minterms just describe full state vectors of the plant. Let $s$ be the formula associated with the Top-Event of of a fault tree, then products $\pi$ that satisfy $s(\pi \in s)$ are the cutsets of $s$.

Let $s$ be a Boolean function and $\mathcal{L}=\left\{L_{1}, \ldots, L_{k}\right\}$ be a set of literals built over a subset of $\operatorname{var}(s)$. We denote by $s \mid \mathcal{L}$ the Boolean function built over 
$\operatorname{var}(s) \backslash \operatorname{var}(\mathcal{L})$ as follows.

$$
s \mid\left\{L_{1}, \ldots, L_{k}\right\} \stackrel{\text { def }}{=}\left\{\pi \mid L_{1} \ldots . L_{k} . \pi \in s\right\}
$$

For the sake of the simplicity, we write $s \mid L_{1}, \ldots, L_{k}$ (instead of $s \mid\left\{L_{1}, \ldots, L_{k}\right\}$ ). The notation $s \mid L$ is intentionally close to the one used for conditional probabilities because it is really what it means: $s$ given $L$.

Example $(s \mid L)$ : Considering the function $s_{1}$ defined above, the following equalities hold.

$$
\begin{aligned}
s_{1} \mid A & \equiv B \cdot C+B \cdot \bar{C}+\bar{B} \cdot C=B+C \\
s_{1} \mid \bar{A} & \equiv B . C \\
s_{1} \mid A, B & \equiv C \\
s_{1} \mid A, \bar{B} & \equiv C
\end{aligned}
$$

\subsection{Shannon Decomposition and Coherence}

We can now state the Shannon decomposition.

Property 1 ((Logical) Shannon Decomposition) Let $s$ be a Boolean formula and $E$ a Basic Event of var(s). Then, the following equivalence holds.

$$
s \equiv E . s|E+\bar{E} . s| \bar{E}
$$

The Shannon decomposition is often called the Pivotal Decomposition in the Reliability Engineering literature.

Throughout this article, we shall assume that Basic Events are independent from a statistical viewpoint. The above equivalence is translated in terms of probability by the either of the two equalities that will play an important role latter.

Property 2 ((Probabilistic) Shannon Decomposition) Let s be a Boolean formula and $E$ a Basic Event of var(s). Then, the following equalities hold.

$$
\begin{aligned}
& \operatorname{Pr}\{s\}=\operatorname{Pr}\{E\} \cdot \operatorname{Pr}\{s \mid E\}+[1-\operatorname{Pr}\{E\}] \cdot \operatorname{Pr}\{s \mid \bar{E}\} \\
& \operatorname{Pr}\{s\}=\operatorname{Pr}\{E\} \cdot[\operatorname{Pr}\{s \mid E\}-\operatorname{Pr}\{s \mid \bar{E}\}]+\operatorname{Pr}\{s \mid \bar{E}\}
\end{aligned}
$$


In the framework on Safety and Reliability Analyses, Basic Events represent in general failures of components of the system under study. Therefore there is an asymmetry between a positive literal that represents the occurrence of a failure and its negation. The former has in general a much lower probability then the latter.

This asymmetry induces a natural partial order amongst minterms. The minterms $\sigma$ is smaller that the minterms $\pi$, which we denote by $\sigma \sqsubseteq \pi$, if for any Basic Event $E, E \in \sigma$ implies that $E \in \pi$. In other words, all components failed in $\sigma$ are failed in $\pi$ as well.

A Boolean function $s$ is coherent if for any two minterms $\sigma$ and $\pi$ such that $\sigma \sqsubseteq \pi, \sigma \in s$ implies that $\pi \in s$. In other words, in a coherent system, the more there are components failed, the more likely the system itself is failed. Or to put it the other way round, the repair of a component cannot fail the system.

A formula built over only connective "." and "+" is coherent.

Example (Coherence): The above function $s_{1}$ is coherent while $s_{2}$ is not: $\bar{A} \cdot \bar{B} \cdot C \in s_{2}$, but $A \cdot \bar{B} \cdot C \notin s_{2}$.

If a function $s$ is not coherent, it is always possible to consider a coherent upper approximation of $s$. Namely, the coherent envelope of $s$, denoted as $\lceil s\rceil$, which is the smallest coherent set of minterms that contains $s$.

$$
\lceil s\rceil \stackrel{\text { def }}{=}\{\pi \in \operatorname{Minterms}(\operatorname{var}(s)) ; \exists \sigma, \sigma \sqsubseteq \pi \wedge \sigma \in s\}
$$

Obviously, for any function $s, s \subseteq\lceil s\rceil$ and $s$ is coherent if and only if $\lceil s\rceil \equiv s$.

Example (Coherence): Consider again $s_{2}=A . B+\bar{A} . C$.

$$
\begin{aligned}
s_{2} & \equiv A \cdot B \cdot C+A \cdot B \cdot \bar{C}+\bar{A} \cdot B \cdot C+\bar{A} \cdot \bar{B} \cdot C \\
\left\lceil s_{2}\right\rceil & \equiv A \cdot B \cdot C+A \cdot B \cdot \bar{C}+A \cdot \bar{B} \cdot C+\bar{A} \cdot B \cdot C+\bar{A} \cdot \bar{B} \cdot C \\
& \equiv A \cdot B+C
\end{aligned}
$$

The added minterm is boxed. 


\subsection{Critical States}

The notion of Critical State is the core of the theory of Importance Measures. Although this notion may sound familiar to the reader, to our knowledge, the presentation we give here is original and completes an interpretation sketched by Vaurio in Reference [22].

Let $s$ be Boolean function and $L$ be literal built over a basic event of $\operatorname{var}(s)$. We define Critical and Non-Critical States of $s$ with respect to $L$ as follows.

$$
\begin{aligned}
& \operatorname{crit}(s, L) \stackrel{\text { def }}{=}\{L . \pi \in s ; \bar{L} . \pi \in \bar{s}\} \\
& \overline{\operatorname{crit}}(s, L) \stackrel{\text { def }}{=}\{L . \pi \in s ; \bar{L} . \pi \in s\}
\end{aligned}
$$

Intuitively, a minterm $L . \pi$ of $s$ is critical with respect to the literal $L$ if by flipping the value of $L$ we change also the value of $s$.

Example (Critical States): Consider again the function $s_{1}=A . B+A . C+$ B.C. We have:

$$
\begin{aligned}
& \operatorname{crit}\left(s_{1}, A\right)=A \cdot B \cdot \bar{C}+A \cdot \bar{B} \cdot C \\
& \overline{\operatorname{crit}}\left(s_{1}, A\right)=A \cdot B \cdot C \\
& \operatorname{crit}\left(s_{1}, \bar{A}\right)=0 \\
& \overline{\operatorname{crit}}\left(s_{1}, \bar{A}\right)=\bar{A} \cdot B \cdot C \\
& \operatorname{crit}\left(\overline{s_{1}}, A\right)=0 \\
& \overline{\operatorname{crit}}\left(\overline{s_{1}}, A\right)=A \cdot \bar{B} \cdot \bar{C} \\
& \operatorname{crit}\left(\overline{s_{1}}, \bar{A}\right)=\bar{A} \cdot B \cdot \bar{C}+\bar{A} \cdot \bar{B} \cdot C \\
& \overline{\operatorname{crit}}\left(\overline{s_{1}}, \bar{A}\right)=\bar{A} \cdot \bar{B} \cdot \bar{C}
\end{aligned}
$$

As suggested by the above example, the notion of criticality separates the minterm space into 8 zones, according to the 3 binary choices $s / \bar{s}, E / \bar{E}$ and Critical/Critical. This separation is graphically illustrated on Figure 1.

This figure illustrates also two simple properties (that follows immediately from the definitions):

$$
\begin{aligned}
& E . s=\operatorname{crit}(s, E)+\overline{\operatorname{crit}}(s, E) \\
& \bar{E} . s=\operatorname{crit}(s, \bar{E})+\overline{\operatorname{crit}}(s, \bar{E})
\end{aligned}
$$




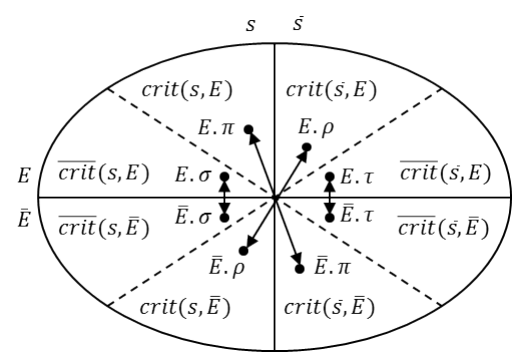

Fig. 1. Minterm Zones

Moreover, zones are paired by flipping the value of $E$, as established by the following property.

Property 3 (Minterms Zones) Let $s$ be a Boolean function and $E$ be a Basic Event of var(s). Then, the following equivalences hold.

$$
\begin{aligned}
& \operatorname{crit}(s, E)|E \equiv \operatorname{crit}(\bar{s}, \bar{E})| \bar{E} \\
& \operatorname{crit}(s, \bar{E})|\bar{E} \equiv \operatorname{crit}(\bar{s}, E)| E \\
& \overline{\operatorname{crit}}(s, E)|E \equiv \overline{\operatorname{crit}}(s, \bar{E})| \bar{E} \\
& \overline{\operatorname{crit}}(\bar{s}, E)|E \equiv \overline{\operatorname{crit}}(\bar{s}, \bar{E})| \bar{E}
\end{aligned}
$$

Intuitively, the above property says that for any minterm $E . \pi$ of $\operatorname{crit}(s, E)$ the minterm $\bar{E} . \pi$ belongs to $\operatorname{crit}(\bar{s}, \bar{E})$ and vice-versa.

The above logical equivalences translate indeed into probabilities: $\operatorname{Pr}\{\operatorname{crit}(s, E) \mid E\}=$ $\operatorname{Pr}\{\operatorname{crit}(\bar{s}, \bar{E}) \mid \bar{E}\}, \ldots$

In the case $s$ represents a coherent system, the repair of a component cannot fail the system. Therefore, the following property holds.

Property 4 (Minterms Zones of Coherent Systems) Let $s$ be a coherent Boolean function and $E$ be a Basic Event of var(s). Then, the following equalities hold.

$$
\begin{aligned}
\operatorname{crit}(s, \bar{E}) & =\operatorname{crit}(\bar{s}, E)=0 \\
\overline{\operatorname{crit}}(s, E) \mid E & =\overline{\operatorname{crit}}(s, \bar{E})|\bar{E}=s| \bar{E}
\end{aligned}
$$

We have now all the elements to revisit Importance Measures. 


\section{Importance Measures of Simple Components}

Importance Measures have been introduced in the seventies (e.g. [23-25]), i.e. at a time where the predominant, if not the only, technology to assess Fault Trees consisted in calculating probabilistic measures from Minimal Cutsets (see e.g. [26,27]). For this reason, most of Importance Measures are usually defined and calculated in terms of Minimal Cutsets. In the nineties, a new assessment technology for Fault Trees came into the play: Bryant's Binary Decision Diagrams $[28,29]$ (BDD for short). To the noticeable exception of large models of the Nuclear Industry, BDD have proved to outperform the Minimal Cutsets technology (see e.g. [30] an overview of their use for risk analyses). Amongst other advantages over the Minimal Cutsets approach, BDD make it possible to calculate exact values of probabilistic measures for they encode sets of Minterms. In 2001, the authors published an article in which they proposed BDD based algorithms to calculate Importance Measures [13] (a first step into this direction has been done before by Andrews in [31]). To do so, we derived "pure" mathematical definitions from actual definitions of Importance Measures (in terms of Minimal Cutsets), therefore separating mathematical concepts from calculation means. We follow here the same line (a full discussion about the drawbacks of definitions of Importance Measures in terms of Minimal Cutsets or Prime Implicants can be found in [?]).

\subsection{Birnbaum Importance Measure}

In many scientific domains, parametric models are defined and a central question is to measure the sensitivity of the model to variations of its parameters. A way to do so is the so-called Marginal Gain technique which consists in making each of the parameter vary slightly in turn, and to observe, mutatis mutandis, the variations induced on the measure(s) at stake.

This typical local sensitivity technique has been used by Birnbaum in [23] to express the importance measure he proposed. The Birnbaum Importance Measure ( $I_{B}$ for short), denoted by $\operatorname{BIM}(s, E)$, is defined as follows.

$$
\operatorname{BIM}(s, E) \stackrel{\text { def }}{=} \frac{\partial[\operatorname{Pr}\{s\}]}{\partial[\operatorname{Pr}\{E\}]}
$$

The Birnbaum Importance Measure can be assessed by calculating first $\operatorname{Pr}\{s\}$ with the regular value of $\operatorname{Pr}\{E\}$, then with a slightly modified value of $\operatorname{Pr}\{E\}$, e.g. $\operatorname{Pr}\{E\}+\epsilon$. But it is general more efficient and anyway more interesting to apply the second form of the Shannon decomposition (eq. 2): 


$$
\begin{aligned}
\operatorname{BIM}(s, E) & \stackrel{\text { def }}{=} \frac{\partial[\operatorname{Pr}\{s\}]}{\partial[\operatorname{Pr}\{E\}]} \\
& =\frac{\partial[\operatorname{Pr}\{E\} \cdot[\operatorname{Pr}\{s \mid E\}-\operatorname{Pr}\{s \mid \bar{E}\}]-\operatorname{Pr}\{s \mid \bar{E}\}]}{\partial[\operatorname{Pr}\{E\}]} \\
& =\operatorname{Pr}\{s \mid E\}-\operatorname{Pr}\{s \mid \bar{E}\}
\end{aligned}
$$

Now look at Figure 1. When $s$ is coherent, according to property $4, \operatorname{crit}(s, \bar{E})=$ 0 . Therefore, according to property $3, \operatorname{Pr}\{s \mid \bar{E}\}=\overline{\operatorname{crit}}(s, \bar{E})|\bar{E}=\overline{\operatorname{crit}}(s, E)| E$. Since $s|E=s \cdot E| E=(\operatorname{crit}(s, E)+\overline{\operatorname{crit}}(s, E)) \mid E$, the following property holds.

Property 5 (Birnbaum Importance Measure and Critical States) Let $s$ be a coherent Fault Tree and $E$ be a Basic Event of $s$, then the following equalities hold.

$$
\begin{aligned}
& \operatorname{BIM}(s, E)=\operatorname{Pr}\{\operatorname{crit}(s, E) \mid E\} \\
& \operatorname{BIM}(s, E)=\operatorname{Pr}\{\operatorname{crit}(s, E)\}+\operatorname{Pr}\{\operatorname{crit}(\bar{s}, \bar{E})\}
\end{aligned}
$$

As we shall see, equations 4 and 5 play a central role in Importance Measures theory.

\subsection{Criticality Importance Measure}

One on the inconvenient of the Birnbaum Importance Measure is that it does not take into account the probability of the Basic Event. So, if two Basic Events play similar roles their ranking according to the Birnbaum Importance Measure will be close, even if their probabilities differ by orders. The Criticality Importance Measure (CIM for short), denoted by $\operatorname{CIM}(s, E)$, is an attempt to correct this drawback. It has been introduced by Lambert [24]. It is defined as follows.

$$
\operatorname{CIM}(s, E) \stackrel{\text { def }}{=} \frac{\operatorname{Pr}\{E\} \times \operatorname{BIM}(s, E)}{\operatorname{Pr}\{s\}}
$$

By equality $4, \operatorname{Pr}\{E\} \times \operatorname{BIM}(s, E)=\operatorname{Pr}\{\operatorname{crit}(s, E)\}$. Moreover the denominator $\operatorname{Pr}\{s\}$ will be the same for all Basic Events and therefore play no role in the raking of components of a same model. It should be seen as a way to normalize results over different models. $\operatorname{CIM}(s, E)$ is directly a measure of criticality of the component $E$. 


\subsection{Diagnostic Importance Measure}

The Diagnostic Importance Measure does not attempt to measure the criticality of components but rather to determine which component should be looked at first when the system is failed in order to repair it. This notion is of interest in harsh environments where sending a robot, or even worse a human operator, may present serious difficulties. So the faster one finds the problem, the better.

The Diagnostic Importance Measure, denoted by $\operatorname{FVM}(s, E)$, has been introduced by Fussel and Vesely in Reference [25] (we name it here after its authors). It is defined as follows.

$$
\operatorname{FVM}(s, E) \stackrel{\text { def }}{=} \operatorname{Pr}\{E \mid s\}
$$

By conditional probability rule, the following equality holds.

$$
\operatorname{FVM}(s, E)=\frac{\operatorname{Pr}\{E . s\}}{\operatorname{Pr}\{s\}}
$$

In other words, $\operatorname{FVM}(s, E)$ is the fraction of the system unavailability (or risk) that involves the component failure. Again $\operatorname{Pr}\{s\}$ should be seen as a normalization factor.

\subsection{Risk Achievement Worth and Risk Reduction Worth}

The two other main Importance Measures, widely used in Nuclear PSA, are the Risk Achievement Worth (RAW for short) and Risk Reduction Worth (RRW for short) (also called respectively Risk Increase Factor and Risk Decrease Factor [32]). They are defined as follows.

$$
\begin{aligned}
& \operatorname{RAW}(s, E) \stackrel{\text { def }}{=} \frac{\operatorname{Pr}\{s \mid E\}}{\operatorname{Pr}\{s\}} \\
& \operatorname{RRW}(s, E) \stackrel{\text { def }}{=} \frac{\operatorname{Pr}\{s \mid \bar{E}\}}{\operatorname{Pr}\{s\}}
\end{aligned}
$$

RAW measures the increase in system failure probability assuming the worst case of failing component. It is an indicator of the importance of maintaining 
the current level of reliability for the component [6]. In reference [4], it is argued that RAW should be used with care, for it is rather rough.

RRW represents the maximum decreasing of the risk it may be expected by increasing the reliability of the component. Therefore this quantity may be used to select components that are the best candidates for efforts leading to improving system reliability. Note that RRW is sometimes defined as $\frac{\operatorname{Pr}\{s\}}{\operatorname{Pr}\{s \mid \bar{E}\}}$, i.e. the inverse of the above definition (e.g. in RiskSpectrum [32]). Taking one definition or the other does change anything but the presentation of the results.

None of these Importance Measures take into account the probability of failure of the component and both are normalized with the denominator $\operatorname{Pr}\{s\}$.

\subsection{Discussion}

At this point, we can organize Importance Measures for coherent systems according to the set of minterms (or states) they intend to capture. These sets are illustrated Figure 2.

- The states in which both the component and the system are failed, as for the FVM and the RAW (Figure 2 (a)).

- The states in which the system is failed but the component is working, as for the RRW (Figure 2 (b)).

- The critical failed states, as for the CIM (Figure 2 (c)) and the critical failed and working states as for the BIM (Figure $2(\mathrm{~d})$ ).

There are two other axes to classify Importance Measures:

- Whether they are normalized using $\operatorname{Pr}\{s\}$ as a denominator, as for the CIM, the FVM, the RAW and the RRW.

- Whether they take into account the probability of failure of the components, as for the CIM and the FVM.

\section{Importance Measures for Complex Components}

In the previous section, we assumed that a component has a single failure mode, represented by a Basic Event. We shall examine now the case where the component is complex, i.e. that its failures are represented by means of a formula. Such an extension is of interest for at least two reasons. First, as pointed out for instance in Reference [33], it is in general difficult when 


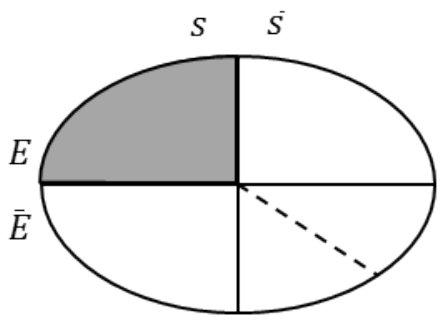

(a) E.S

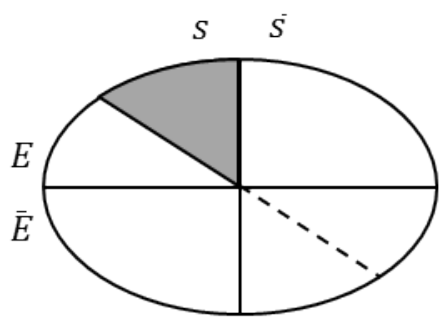

(c) $\operatorname{crit}(s, E)$

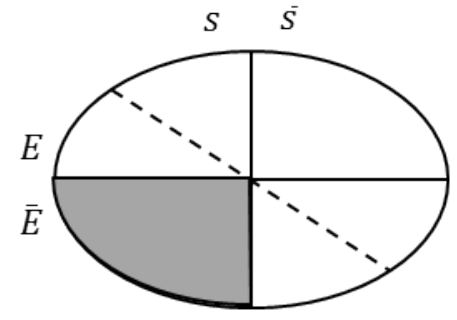

(b) $\bar{E} . S$

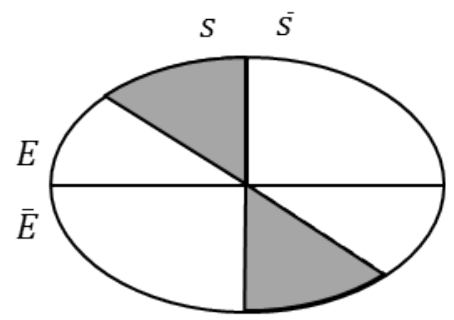

(d) $\operatorname{crit}(s, E)+\operatorname{crit}(\bar{s}, \bar{E})$

Fig. 2. Minterms set described by Importance Measures

evaluating the consequences of a change in maintenance and operation policy to assume a complete independence of events. Therefore considering only individual contributions to the risk is often insufficient. With that respect, the extension of Importance Measures to groups of components is necessary. Cheok \& al. [6] give Motor Operated Valves as a typical example of such a group in Nuclear Power Plants. Second, whether a component is considered as atomic or decomposed is to some extent a choice of the analyst. Therefore, it should be possible to calculate seamlessly the same measure whether the component is atomic or complex.

In their article [6], Cheok and al. studied thoroughly the problems raised by the extension of Importance Measures to groups of Basic Events. We show here that these problems are deeply rooted from an algebraic viewpoint and we propose some solutions.

\subsection{Risk Achievement Worth and Risk Reduction Worth}

In the sequel, we shall assume that the failures of the system and the component are modeled respectively by a coherent function $s$ and a coherent function c. Moreover, we assume without a loss of generality that $\operatorname{var}(c) \subseteq \operatorname{var}(s)$ and that $c$ is not a module of $s$, i.e. it shares some Basic Events with other parts (gates) of $s$.

We can first remark that it is still possible to calculate conditional probabilities $\operatorname{Pr}\{s \mid c\}$ and $\operatorname{Pr}\{s \mid \bar{c}\}$ using the central property of conditional probabilities. 


$$
\begin{aligned}
\operatorname{Pr}\{s \mid c\} & =\frac{\operatorname{Pr}\{s . c\}}{\operatorname{Pr}\{c\}} \\
\operatorname{Pr}\{s \mid \bar{s}\} & =\frac{\operatorname{Pr}\{s . \bar{c}\}}{1-\operatorname{Pr}\{c\}}
\end{aligned}
$$

These calculations may require a significant overhead because they require building a Binary Decision Diagram or extracting Minimal Cutsets of s.c and $s . \bar{c}$ (or at least one of those for $\operatorname{Pr}\{s . \bar{c}\}=\operatorname{Pr}\{s\}-\operatorname{Pr}\{s . c\}$ ). Moreover, it is not possible to interpret $\operatorname{Pr}\{s \mid c\}$ and $\operatorname{Pr}\{s \mid \bar{c}\}$ as the probabilities of some sets of minterms. As an illustration, consider the following example.

Example $(\operatorname{Pr}\{s \mid c\})$ : Consider again the formula $s=A . B+A . C+B . C$ and let $c=A$. B. Assume that $\operatorname{Pr}\{A\}=\operatorname{Pr}\{B\}=\operatorname{Pr}\{C\}=1 / 2$ so that all minterms have the probability $1 / 8$. The following equalities hold.

$$
\begin{aligned}
& \operatorname{Pr}\{s\}=\operatorname{Pr}\{A \cdot B \cdot C+A \cdot B \cdot \bar{C}+A \cdot \bar{B} \cdot C+\bar{A} \cdot B \cdot C\} \\
& =4 / 8 \\
& \operatorname{Pr}\{c\}=\operatorname{Pr}\{A \cdot B \cdot C+A \cdot B \cdot \bar{C}\}=2 / 8 \\
& \operatorname{Pr}\{\bar{c}\}=1-\operatorname{Pr}\{c\}=6 / 8 \\
& \operatorname{Pr}\{\text { s.c }\}=\operatorname{Pr}\{A \cdot B . C+A \cdot B \cdot \bar{C}\}=2 / 8 \\
& \operatorname{Pr}\{s \cdot \bar{c}\}=\operatorname{Pr}\{A \cdot \bar{B} \cdot C+\bar{A} \cdot B \cdot C\}=2 / 8 \\
& \operatorname{Pr}\{s \mid c\}=\quad \frac{2 / 8}{2 / 8} \quad=1 \\
& \operatorname{Pr}\{s \mid \bar{s}\}=\quad \frac{2 / 8}{6 / 8} \quad=1 / 3
\end{aligned}
$$

Since $1 / 3$ is prime with $1 / 8, \operatorname{Pr}\{s \mid \bar{c}\}$ cannot be interpreted as the probability of set of minterms.

Note however that this does not prevent to keep as such the definition of the Diagnostic Importance Measure.

Bearing that in mind, we can attempt to keep also the same definitions for the Risk Achievement Worth and the Risk Reduction Worth:

$$
\begin{aligned}
& \operatorname{FVM}_{P r}(s, c) \stackrel{\text { def }}{=} \operatorname{Pr}\{c \mid s\}=\frac{\operatorname{Pr}\{s . c\}}{\operatorname{Pr}\{s\}} \\
& \operatorname{RAW}_{P r}(s, c) \stackrel{\text { def }}{=} \frac{\operatorname{Pr}\{s \mid c\}}{\operatorname{Pr}\{s\}}=\frac{\operatorname{Pr}\{s . c\}}{\operatorname{Pr}\{c\} \times \operatorname{Pr}\{s\}} \\
& \operatorname{RRW}_{P r}(s, c) \stackrel{\text { def }}{=} \frac{\operatorname{Pr}\{s \mid \bar{c}\}}{\operatorname{Pr}\{s\}}=\frac{\operatorname{Pr}\{s . \bar{c}\}}{(1-\operatorname{Pr}\{c\}) \times \operatorname{Pr}\{s\}}
\end{aligned}
$$

Note first that it may be better to characterize states in which the component 
is failed (respectively working) by using straight $\operatorname{Pr}\{s . c\}$ and $\operatorname{Pr}\{s . \bar{c}\}$, possibly normalized with $\operatorname{Pr}\{s\}$, rather than $\mathrm{RAW}_{P r}(s, c)$ and $\mathrm{RRW}_{P r}(s, c)$ that do not characterize any minterm set.

Moreover, there is another issue here: the Risk Reduction Worth aims to capture the maximum decrease of risk one gets by ensuring that component is working. But what does it mean in the case of a complex component? The above definition just asserts that the component is working, no matter how. As an alternative, we can consider that the component is as good as new. Symmetrically (for the Risk Achievement Worth), we can consider that the component is completely failed rather than just failed no matter how. These extreme cases would lead to the following alternative definitions for these indicators.

$$
\begin{aligned}
& \operatorname{RAW}_{B E}(s, c) \stackrel{\text { def }}{=} \frac{\operatorname{Pr}\{s \mid \operatorname{var}(c)\}}{\operatorname{Pr}\{s\}} \\
& \operatorname{RRW}_{B E}(s, c) \stackrel{\text { def }}{=} \frac{\operatorname{Pr}\{s \mid \overline{\operatorname{var}(c)}\}}{\operatorname{Pr}\{s\}}
\end{aligned}
$$

where $s \mid \operatorname{var}(c)$ and $s \mid \overline{\operatorname{var}(c)}$ denote respectively the function $s$ with all Basic Events of $c$ set to 1 and to 0 (hence the index BE).

Indicators $\mathrm{RAW}_{B E}(s, c)$ and $\mathrm{RRW}_{B E}(s, c)$ are coarse in this sense that they treat in the same way components with different structure functions. As we shall see in the next section however, they are used by most of the authors (and software tools) to handle groups of Basic Events. Moreover, they do characterize sets of minterms, which a priori eases to give them a physical interpretation.

Since both $s$ and $c$ are coherent, the following inequalities hold.

$$
\begin{aligned}
& \operatorname{Pr}\{s \mid c\} \leq \operatorname{Pr}\{s \mid \operatorname{var}(c)\} \\
& \operatorname{Pr}\{s \mid \bar{c}\} \geq \operatorname{Pr}\{s \mid \overline{\operatorname{var}(c)}\}
\end{aligned}
$$

which translates immediately into the following inequalities.

$$
\begin{aligned}
& \mathrm{RAW}_{P r}(s, c) \leq \operatorname{RAW}_{B E}(s, c) \\
& \operatorname{RRW}_{P r}(s, c) \geq \operatorname{RRW}_{B E}(s, c)
\end{aligned}
$$




\subsection{Birnbaum Importance Measure}

According to section 3.5 and the above discussion, we have the three following candidate definitions for the (extended) Birnbaum Importance Measure.

$$
\begin{aligned}
\operatorname{BIM}_{\partial}(s, c) & \stackrel{\text { def }}{=} \frac{\partial[\operatorname{Pr}\{s\}]}{\partial[\operatorname{Pr}\{c\}]} \\
\operatorname{BIM}_{P r}(s, c) & \stackrel{\text { def }}{=} \operatorname{Pr}\{s \mid c\}-\operatorname{Pr}\{s \mid \bar{c}\} \\
\operatorname{BIM}_{B E}(s, c) & \stackrel{\text { def }}{=} \operatorname{Pr}\{s \mid \operatorname{var}(c)\}-\operatorname{Pr}\{s \mid \overline{\operatorname{var}(c)}\}
\end{aligned}
$$

The indicator $\operatorname{BIM}_{\partial}(s, c)$ is problematic. If the component $c$ shares Basic Events with other parts of the model, it is not possible to make the probability of $c$ vary mutatis mutandis, i.e. without impacting the probability of these parts. That is the reason why some authors reject it purely and simply (e.g. [20]). Moreover, since $c$ depends on several Basic Events, one should determine how the probabilities of these Basic Events must vary in order to make $\operatorname{Pr}\{c\}$ vary. In a word, it is not clear how to calculate $\operatorname{BIM}_{\partial}(s, c)$.

For this reason, several authors (e.g. [21]) suggested to take $\operatorname{BIM}_{P r}(s, c)$ as the reference, leaving however open the question of its physical interpretation, as illustrated by the example of the previous section.

In her diploma thesis [14], Sutter proposed to use $\operatorname{BIM}_{B E}(s, c)$ for complex components. Again, this definition is coarse for it gives the same results for components with different structure functions but at least it does characterize a set of minterms. Since since $s$ is monotone, we we have $\operatorname{Pr}\{s \mid \overline{\operatorname{var}(c)}\} \subseteq$ $\operatorname{Pr}\{s \mid \operatorname{var}(c)\}$. Therefore the following equality holds.

$$
\operatorname{Pr}\{s \mid \operatorname{var}(c)\}-\operatorname{Pr}\{s \mid \overline{\operatorname{var}(c)}\}=\operatorname{Pr}\{s \mid \operatorname{var}(c) \cdot \overline{s \mid \overline{\operatorname{var}(c)}}\}
$$

The following example illustrates this equality.

Example (Set of minterms characterized by $\operatorname{BIM}_{B E}(s, c)$ ): Let $s=$ $A . B+A . C+C . D$ and $c=A . B$. Then $s|\operatorname{var}(c)=s| A, B=C$ and $s \mid \overline{\operatorname{var}(c)}=$ $s \mid \bar{A}, \bar{B}=C . D$. Therefore $\operatorname{BIM}_{B E}(s, c)=\operatorname{Pr}\{C . \overline{C . D}\}=\operatorname{Pr}\{C . \bar{D}\}$.

\subsection{Critical States}

In section 3.1, we recalled that the Birnbaum Importance Measure is strongly related to the notion of critical state. Namely, according to equality 5 , the 
Birnbaum Importance Measure is the probability to be either in a critical failure state or in a critical working state w.r.t. the component (see e.g. [16,34]). In the case of a simple component, this definition/property is clear. In the case of a complex component, the above idea gives rise to three alternative definitions of critical failure states. Before introducing these definitions, we need some additional notations.

Let $\sigma$ be a minterm built over a set $\mathrm{E}$ of Basic Events and let $\mathrm{G}$ be a subset of E. We denote by $\sigma \downarrow \mathcal{G}$, respectively $\sigma \uparrow \mathcal{G}$, the minterm such that for any Basic Event $E \sigma \downarrow \mathcal{G}(E)=0$, respectively $\sigma \uparrow \mathcal{G}(E)=1$, if $E \in \mathcal{G}$ and $\sigma(E)$ otherwise.

Now, a state is a critical failure state if:

(1) The system is failed in that state and there is a mean to repair it by repairing some of the constituents of the component. Since the system is coherent, this is equivalent to say that the system is repaired by repairing all of the constituents of the component. Formally:

$$
\operatorname{crit}_{f, B E}(s, c) \stackrel{\text { def }}{=}\{\pi \in s ; \pi \downarrow \operatorname{var}(c) \in \bar{s}\}
$$

(2) Both the system and the component are failed in that state and there is a mean to repair the system by repairing the component. Formally:

$$
\operatorname{crit}_{f, \exists}(s, c) \stackrel{\text { def }}{=}\{\pi \in s . c ; \pi \downarrow \operatorname{var}(c) \in \bar{s}\}
$$

(3) Both the system and the component are failed in that state and any repair of the component repairs the system. Formally:

$$
\operatorname{crit}_{f, \forall}(s, c) \stackrel{\text { def }}{=}\{\pi \in s . c ; \forall \sigma \sqsubset \pi, \sigma \in \bar{c} \Rightarrow \sigma \in \bar{s}\}
$$

Symmetrically, there are three alternative definitions of critical working state. A state is a critical working state if:

(1) The system is working in that state and there is a mean to fail it by failing some of the constituents of the component. Formally:

$$
\operatorname{crit}_{w, B E}(s, c) \stackrel{\text { def }}{=}\{\pi \in \bar{s} ; \pi \uparrow \operatorname{var}(c) \in s\}
$$

(2) Both the system and the component are working in that state and there is a mean to repair the system by failing the component. Formally:

$$
\operatorname{crit}_{w, \exists}(s, c) \stackrel{\text { def }}{=}\{\pi \in \bar{s} . \bar{c} ; \pi \uparrow \operatorname{var}(c) \in s\}
$$

(3) Both the system and the component are working in that state and any failure of the component fails the system. Formally: 


$$
\operatorname{crit}_{w, \forall}(s, c) \stackrel{\text { def }}{=}\{\pi \in \bar{s} . \bar{c} ; \forall \sigma \sqsupset \pi, \sigma \in c \Rightarrow \sigma \in s\}
$$

The three definitions are ordered. Namely, the following inclusions hold.

$$
\begin{aligned}
\operatorname{crit}_{f, \forall}(s, c) \subseteq \operatorname{crit}_{f, \exists}(s, c) \subseteq \operatorname{crit}_{f, B E}(s, c) \\
\operatorname{crit}_{w, \forall}(s, c) \subseteq \operatorname{crit}_{w, \exists}(s, c) \subseteq \operatorname{crit}_{w, B E}(s, c)
\end{aligned}
$$

The result follows immediately from the definitions. The inclusions can be strict, as shown by the following example.

Example (Strict Inclusion of the three definitions Critical States): Let $s=A . B . C+A . D$ and $c=A . B$. We have:

$$
\begin{aligned}
\operatorname{crit}_{f, B E}(s, c)= & A \cdot B \cdot C \cdot D+A \cdot B \cdot C \cdot \bar{D}+A \cdot B \cdot \bar{C} \cdot D+ \\
& A \cdot \bar{B} \cdot C \cdot D+A \cdot \bar{B} \cdot \bar{C} \cdot D \\
\operatorname{crit}_{f, \exists}(s, c)= & A \cdot B \cdot C \cdot D+A \cdot B \cdot C \cdot \bar{D}+A \cdot B \cdot \bar{C} \cdot D \\
\operatorname{crit}_{f, \forall}(s, c)= & A \cdot B \cdot C \cdot \bar{D}
\end{aligned}
$$

The above example illustrates a quite surprising point: $\operatorname{crit}_{f, B E}(s, c)$ contains states in which the system is failed but the component is not (e.g. A.B.C.D). The introduction of $\operatorname{crit}_{f, B E}(s, c)$ and $\operatorname{crit}_{w, B E}(s, c)$ is however justified by the following equality.

$$
s \mid \operatorname{var}(c) . \overline{s \mid \overline{\operatorname{var}(c)}}=\operatorname{crit}_{f, B E}(s, c)+\operatorname{crit}_{w, B E}(s, c)
$$

The proof is done by showing the inclusion in both directions.

The following property is directly derived from equality 6 .

\section{Property 6 ((Extended) Birnbaum Importance Measure and Critical States)}

Let $s$ and $c$ be two coherent Fault Trees $(\operatorname{var}(c) \subseteq \operatorname{var}(s))$. Then the following equality holds.

$$
B I M_{B E}(s, c)=\operatorname{Pr}\left\{\operatorname{crit}_{f, B E}(s, c)\right\}+\operatorname{Pr}\left\{\operatorname{crit}_{w, B E}(s, c)\right\}
$$

The above equality is a sound generalization of equality 5 , up to a quite high price: first, one is not able to distinguish components with different structure functions, and second one should consider as critical failure (resp. working) states, states in which the component is not failed (resp. working). This problem has been so far unnoticed in the literature. 
The indicator $\operatorname{crit}_{f, \exists}(s, c)$ does not solve the first problem, but it solves the second one. It has probably only little practical value. However, it was important to introduce it here to make a bridge between definitions of $\operatorname{crit}_{f, B E}(s, c)$ and $\operatorname{crit}_{f, \forall}(s, c)$.

The indicator $\operatorname{crit}_{f, \forall}(s, c)$ solves both problems. To our feeling, it is the one that corresponds the best to the intuitive notion of critical states. It could serve as a valuable alternative definition for the Birnbaum Importance Measure:

$$
\operatorname{BIM}_{\forall}(s, c) \stackrel{\text { def }}{=} \operatorname{Pr}\left\{\operatorname{crit}_{f, \forall}(s, c)\right\}+\operatorname{Pr}\left\{\operatorname{crit}_{w, \forall}(s, c)\right\}
$$

This indicator may be however tedious to calculate.

To finish this section, let us remark that all alternative definitions for the Birnbaum Importance Measure induce as many alternative definitions for the Criticality Importance Measure and that they all agree in the case the component is simple.

\subsection{Groups of Components}

Assessing the risk significance of a group of components that play a similar role, e.g. valves or pumps, raises similar difficulties even if we assume that the components in question are simple ones (i.e. represented by Basic Events), see e.g. [6]. It would be convenient to assimilate the group as a complex component. But which Boolean function should be used to gather failures of these components? Should these failures and-ed, or-ed? Should the formula depend on the calculated measure?

In Risk Spectrum [32] for instance, the Risk Achievement Worth and Risk Reduction Worth of a group $\mathcal{G}$ of Basic Events are defined as $\operatorname{RAW}_{B E}(s, \mathcal{G})$ and $\operatorname{RRW}_{B E}(s, \mathcal{G})$.

Regarding the Birnbaum Importance Measure (and thus the Criticality Importance Measure), all of the authors we know (e.g. $[15-17,14,12])$ take $\operatorname{BIM}_{B E}(s, \mathcal{G})$ as reference.

Another idea has been proposed by Borgonovo \& al. [35,36] and Lemaire [37] independently. It consists in defining an additive measure, i.e. a measure such as the contribution of a group is the sum of the individual contributions of its components. The Differential Importance Measure (DIM for short) is defined as follows. 


$$
\operatorname{DIM}(s, \mathcal{G}) \stackrel{\text { def }}{=} \frac{\sum_{E_{i} \in \mathcal{G}} \frac{\partial \operatorname{Pr} s}{\partial \operatorname{Pr}\left\{E_{i}\right\}} d \operatorname{Pr}\left(E_{i}\right)}{\sum_{E_{i} \in \mathcal{E}} \frac{\partial \operatorname{Pr} s}{\partial \operatorname{Pr}\left\{E_{i}\right\}} d \operatorname{Pr}\left(E_{i}\right)}
$$

In the case we consider a uniform change of the $\operatorname{Pr} E_{i}$ 's $\left(d \operatorname{Pr} E_{i}=d \operatorname{Pr} E_{j}\right.$ for all $i$ and $j$ ), this definition can be instantiated as follows [10].

$$
\operatorname{DIM}(s, \mathcal{G}) \stackrel{\text { def }}{=} \frac{\sum_{E_{i} \in \mathcal{G}} \operatorname{BIM}\left(s, E_{i}\right)}{\sum_{E_{i} \in \mathcal{E}} \operatorname{BIM}\left(s, E_{i}\right)}
$$

In the case of a proportional changeof the $\operatorname{Pr} E_{i}{ }^{\prime} \mathrm{s}\left(\frac{d \operatorname{Pr} E_{i}}{\operatorname{Pr} E_{i}}=\frac{d \operatorname{Pr} E_{j}}{\operatorname{Pr} E_{j}}\right.$ for all $i$ and $j)$, it can be instianted by replacing in the above equation $\operatorname{BIM}\left(s, E_{i}\right)$ by $\operatorname{CIM}\left(s, E_{i}\right)$.

$\operatorname{DIM}(s, \mathcal{G})$ is clearly additive, i.e. $\operatorname{DIM}(s, \mathcal{G})=\sum_{E_{i} \in \mathcal{G}} \operatorname{DIM}\left(s, \mathcal{E}_{\rangle}\right)$. is the fraction of change in system reliability due to a simultaneous(and related) changes in probabilities of the components of the group. He points out that this measure can be computed without reassessing the model (under the condition that $\operatorname{BIM}\left(s, E_{i}\right)$ 's have been calculated for all Basic Events $E_{i}$ of the system).

Although this measure is of a great interest, it is not possible to give it an interpretation in terms of states of the system, i.e. to define it as the sum of the probabilities of the states of given subset.

The vision in terms of scenarios or global states or minterms, is however of some help here too. When scenarios are of concerned, then analysts might be interested in assessing the probability of following scenarios.

- The scenarios in which the system is failed and at least one component of the group contributes to that failure, i.e. in logical terms $s . \sum_{E_{i} \in G} E_{i}$ or conversely none of the components of group is involved in the failure, i.e. in logical terms $s \cdot \overline{\sum_{E_{i} \in G} E_{i}}$. The Risk Reduction Worth, as defined in Risk Spectrum, measures the probability of this last set (up to the probability of $\left.\overline{\sum_{E_{i} \in G} E_{i}}\right)$.

- The set of states in which repairing one of the component of the group suffices to repair the system, i.e. in logical terms $\operatorname{crit}\left(s, \sum_{E_{i} \in G} E_{i}\right)$.

The scenarios in which all of the components of the group are lost (and the system is lost), i.e. in logical terms $s . \pi_{E_{i} \in G} E_{i}$ may be of interest as well although only in some specific situations. The Risk Achievement Worth as defined in Risk Spectrum, characterizes this last set, again up the probability of $\pi_{E_{i} \in G} E_{i}$. More complex scenarios could be scrutinized as well using other formulas such as $k$-out-of- $n$. 
Example (Group of Components): As an illustration, let $s=g_{1} . g_{2}$, where $g_{i}=\left(A_{i}+B_{i}\right) \cdot C_{i}$ for $i=1,2$. Assume we want to measure the importance of the $A_{i}$ 's, i.e. $G=A_{1}, A_{2}$. Clearly, there are scenarios in which all of the $A_{i}$ are failed and the system is not (if none of the $B_{i}$ 's is failed) and some other where none of the $A_{i}$ 's is failed while the system is (if both the $B_{i}$ 's and the $C_{i}$ 's are failed). There are also scenarios a repair of any of the $A_{i}$ 's repairs the system (e.g. $A_{1} \cdot \overline{B_{1}} \cdot C_{1} \cdot A_{2} \cdot \overline{B_{2}} \cdot C_{2}$ ) and some others where repairing the $A_{i}$ 's has no effect (e.g. $\left.A_{1} \cdot B_{1} \cdot C_{1} \cdot A_{2} \cdot B_{2} \cdot C_{2}\right)$. And so on. If we let $s=2 / 3\left(g_{1}, g_{2}, g_{3}\right)$, the variety of situations even increases and it may be worth to study for instance scenarios in which at least two of the $A_{i}$ 's are failed.

These simple examples show that looking at importance of components in terms of scenarios offers a rich variety of tools to the analyst.

\section{Conclusion}

In this article, we studied the potential extensions of Importance Measures to complex components. We showed that the nice correspondence between the probabilistic definition of these indicators and their minterm interpretation that holds for simple components cannot hold for complex ones. In the literature, this correspondence is somehow artificially maintained for groups of components by considering only the two extreme cases where either all of the components of the group are failed or they are all working. This idea cannot be applied to complex components because it leads to an awkward definition of critical states. The correspondence must therefore be abandoned.

We believe that the framework we propose here is a good starting point to study/revisit extensions of Importance Measures to non-coherent systems (see e.g. $[38,11]$ ), time-dependent systems (see e.g. $[15,39]$ ) or multi-state systems (see e.g. $[40,41])$.

\section{References}

[1] R. S. Andsten, J. K. Vaurio, Sensitivity, uncertainty and importance analysis of a risk assessment, Nuclear Technology 98 (1992) 160-170.

[2] A. Høyland, M. Rausand, System Reliability Theory, John Wiley and Sons, 1994.

[3] F. C. Meng, Some further results on ranking the importance of system components, Reliability Engineering and System Safety 47 (2) (1995) 97-101. 
[4] I. B. Wall, D. H. Worledge, Some perspectives on risk importance measures, in: Proceedings of the international conference on Probabilistic Safety Assessment, PSA'96, 1996, pp. 203-207.

[5] I. N. Kovalenko, N. Y. Kuznetsov, P. A. Pegg, Mathematical Theory of Reliability of Time Dependent Systems with Practical Applications, Wiley Series in Probability and Statistics, John Wiley and Sons, 1997, iSBN 0-47195060-2.

[6] M. C. Cheok, G. W. Parry, R. R. Sherry, Use of importance measures in risk informed regulatory applications, Reliability Engineering and System Safety 60 (3) (1998) 213-226.

[7] M. Modarres, M. Kaminsky, V. Krivstov, Reliability Engineering and Risk Analysis, Marcel Dekker, 1999, iSBN 0-8247-2000-8.

[8] F. C. Meng, Relationships of fussell-vesely and birnbaum importance to structural importance in coherent systems, Reliability Engineering and System Safety 67 (1) (2000) 55-60.

[9] F. C. Meng, Comparing two reliability upper bounds for multistate systems, Reliability Engineering and System Safety 87 (1) (2000) 31-36.

[10] E. Borgonovo, Differential, criticality and birnbaum importance measures: an application to basic event, groups and sscs in event trees and binary decision diagrams, Reliability Engineering and System Safety 92 (2007) 1458-1467.

[11] E. Borgonovo, The reliability importance of components and prime implicants in coherent and non-coherent systems including total-order interactions, European Journal of Operational Research 204 (2010) 485-495.

[12] W. Kuo, X. Zhu, Importance Measures in Reliability, Risk and Optimization Principles and Applications, Wiley, 2012, iSBN 978-1-119-99344-5.

[13] Y. Dutuit, A. Rauzy, Efficient Algorithms to Assess Components and Gates Importances in Fault Tree Analysis, Reliability Engineering and System Safety 72 (2) (2001) 213-222.

[14] E. Sutter, Importance measures in systems which allow repairs, Diploma thesis, University of Bern (2005).

[15] R. E. Barlow, F. Proschan, Importance of system components and fault tree events, Stochastic Processes and their Applications 3 (1975) 153-173.

[16] T. Aven, Reliability and Risk Analysis, Kluwer Academic Publishers, Dordrecht, the Netherlands, 1993.

[17] A. B. Huseby, Importance measure for multicomponent binary systems, Statistical Research Report 11, Dept. of Mathematics, University of Oslo, iSSN 0806-3842 (2004). 
[18] J. K. Vaurio, Developments in importance measures for risk-informed ranking and other applications, in: Proceedings of the 8th International Conference on Probabilistic Safety Assessment and Management (PSAM 8), ASME, New Orleans, 2006.

[19] J. K. Vaurio, Configuration management and maintenance ranking by reliability importance measures, in: C. G. Soares, E. Zio (Eds.), Proceedings of European Safety and Reliability Conference, ESREL'2006, A.A. Balkema, Taylor and Francis Group, Leiden, Estoril, Portugal, 2006, pp. 633-641.

[20] H. S. Lee, C. H. Lie, J. S. Hong, A Computation Method for Evaluating Importance Measures of Gates in Fault Tree, IEEE Transactions on Reliability 46 (3) (1997) 360-365.

[21] J. S. Hong, C. H. Lie, Joint reliability importance of two edges in an undirected network, IEEE Transaction on Reliability 42 (1993) 17-23.

[22] J. K. Vaurio, Ideas and developments of importance measures and fault tree techniques for reliability and risk analysis, Reliability Engineering and System Safety 95 (2) (2010) 95-107.

[23] Z. W. Birnbaum, On the importance of different components and a multicomponent system, in: P. R. Korishnaiah (Ed.), Multivariable analysis II, Academic Press, New York, 1969, pp. 581-592.

[24] H. E. Lambert, Measures of importance of events and cut sets in fault trees, in: R. E. Barlow, J. B. Fussel, N. Singpurwalla (Eds.), Reliability and Fault Tree Analysis, SIAM Press, 1975, pp. 77-100.

[25] J. B. Fussel, How to hand-calculate system reliability characteristics, IEEE Transactions on Reliability R-24 (3) (1975) 169-174.

[26] J. B. Fussel, W. E. Vesely, A New Methodology for Obtaining Cut Sets for Fault Trees, Trans. Am. Nucl. Soc. 15 (1972) 262-263.

[27] J. W. Hickman, A guide to performance of probabilistic risk assessments for nuclear power plants, Tech. Rep. NUREG/CR-2300, US Nuclear Regulatory Commission, Washington D.C. (January 1983).

[28] R. Bryant, Graph Based Algorithms for Boolean Fonction Manipulation, IEEE Transactions on Computers 35 (8) (1986) 677-691.

[29] K. Brace, R. Rudell, R. Bryant, Efficient Implementation of a BDD Package, in: Proceedings of the 27th ACM/IEEE Design Automation Conference, IEEE 0738, 1990, pp. 40-45.

[30] A. Rauzy, BDD for Reliability Studies, in: K. Misra (Ed.), Handbook of Performability Engineering, Elsevier, 2008, pp. 381-396, iSBN 978-1-84800-1305 .

[31] R. Sinnamon, J. Andrews, Improved Accuracy in Qualitative Fault Tree Analysis, Quality and Reliability Engineering International 13 (1997) 285-292. 
[32] U. Berg, RISK SPECTRUM, Theory Manual, RELCON Teknik AB (April 1994).

[33] C. L. Smith, Calculating conditional core damage probabilities for nuclear power plant operations, Reliability Engineering and System Safety 59 (1998) 299-307.

[34] C. Cocozza-Thivent, Processus stochastiques et fiabilité des systèmes, Springer Verlag, 1997, iSBN 3-540-63390-1.

[35] E. Borgonovo, G. E. Apostolakis, A new importance measure for risk-informed decision making, Reliability Engineering and System Safety 72 (2) (2001) 193212.

[36] E. Borgonovo, G. E. Apostolakis, S. Tarantola, A. Saltelli, Comparison of local and global sensitivity analysis techniques in probabilistic safety assessment, Reliability Engineering And System Safety 79 (2) (2003) 175-185.

[37] O. Lemaire, Importance and contribution factor for systems, in: G. Schueller, P. Kafka (Eds.), Proceedings of the ESREL'99 conference, München-Garching, Germany, 1999, pp. 1147-1151.

[38] S. Beeson, J. Andrews, Importance measures for non-coherent-system analysis, IEEE Transactions on Reliability 52 (2003) 301-310.

[39] B. Natvig, A suggestion of a new measure of importance of system components, Stochastic Processes and their Applications 9 (1979) 319-330.

[40] E. Zio, L. Podofillini, Monte-carlo simulation analysis of the effects on different system performance levels on the importance on multi-state components, Reliability Engineering and System Safety 82 (2003) 63-73.

[41] J. K. Vaurio, Importance measures for multi-phase missions, Reliability Engineering and System Safety 99 (1) (2010) 230-235. 